\title{
Mercury: Industrial IBC Cell with Front Floating Emitter For 20.9\% and Higher Efficiency
}

\author{
${ }^{1}$ Agnes Mewe, ${ }^{1}$ Pierpaolo Spinelli, ${ }^{1}$ Antonius Burgers, ${ }^{1}$ Gaby Janssen, ${ }^{1}$ Nicolas Guillevin, ${ }^{2}$ Bas van de Loo, \\ ${ }^{2}$ Erwin Kessels, ${ }^{3}$ Ard Vlooswijk, ${ }^{1}$ Bart Geerligs, and ${ }^{1}$ Ilkay Cesar \\ ${ }^{1}$ ECN Solar Energy, Petten, NL-1755 LE, The Netherlands, ${ }^{2}$ Eindhoven University of Technology, \\ Eindhoven, NL-5612 AP, The Netherlands, ${ }^{3}$ Tempress Systems, Vaassen, NL-8171 MD, The Netherlands
}

\begin{abstract}
In this paper break-through results on our 6" industrial Mercury cells are presented. We gained more than $1 \%$ absolute in efficiency by optimizing the processes and design of the cells, resulting in $\mathbf{2 0 . 9 \%}$ cell efficiency. We used standard industrial equipment and the number of process steps similar to our commercial n-Pasha technology. The screen-printed IBC cells can be interconnected by our proven industrial foil-based interconnection scheme. Together with this result, we present a method to characterize and quantify the pn-junction recombination contribution to $V_{o c}$ and pseudo-FF losses in the cell. Finally, our roadmap to $23 \%$ Mercury cells is presented.

Index Terms - IBC, junction recombination, photovoltaic cells, silicon.
\end{abstract}

\section{INTRODUCTION}

The Mercury cell developed at ECN is an interdigitated back contact (IBC) cell which employs a front floating emitter (FFE). The FFE enables the lateral flow of holes at the front, limiting the effect of electrical shading of the BSF areas at the rear. This lateral flow is referred to as the "pumping effect" of holes, generated above the BSF, through the FFE to the rear emitter [1]. A schematic cross-section of the Mercury cell is shown in Figure 1.

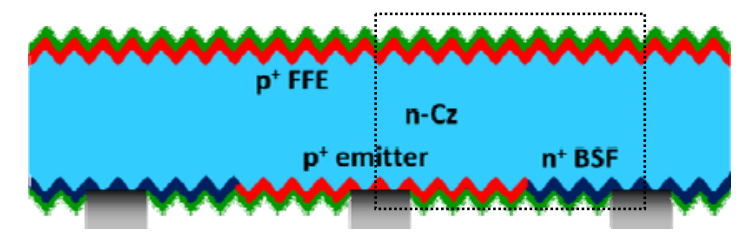

Fig. 1. Schematic cross-section of a Mercury IBC cell. The dashed box indicates the unit cells used in device simulations.

The Mercury cell concept follows an industrial process flow, using the same equipment as for the manufacturing of our industrial n-Pasha cell, and using a similar number of process steps. Cells are processed on commercially available $156 \times 156 \mathrm{~mm}^{2} \mathrm{n}-\mathrm{Cz}$ wafers, and can be readily processed into modules thanks to ECN's foil-based interconnection design, which is a technology that has been proven to pass the IEC 61215 test standards [2].

Recent developments in the process and design of the Mercury cell have resulted in cell efficiencies up to $20.9 \%$ [3], which is an increase in efficiency of more than $1 \%$ absolute compared to the results that were reported previously [4]. In TABLE I, the cell parameters of the best cell are shown. The $I V$ parameters were obtained in an in-house measurement using a class AAA solar simulator. The measurement chuck was especially designed for our Mercury cells, with current and voltage probes only contacting the module interconnection points. The $J_{s c}$ was corrected for spectral mismatch.

The surface passivation of an IBC cell is of high importance, firstly for high $J_{s c}$ due to the long path lengths that minority carriers travel before being collected, and secondly to build up an appreciable carrier density in order to achieve high $V_{o c}$. In this paper we study one particular aspect of the Mercury cell, the pn-junction recombination, which can have a strong impact on cell performance. We present an experimental method to study the passivation of the rear pnjunction, and we elaborate on the physical background to understand it.

TABLE I

SUMMARY OF MERCURY IBC CELl PARAMETERS

\begin{tabular}{|c|c|c|c|c|}
\hline $\begin{array}{c}\text { area } \\
{\left[\mathbf{c m}^{2}\right]}\end{array}$ & $\begin{array}{c}\mathbf{J}_{\text {sc }} \\
{\left[\mathbf{m A} / \mathbf{c m}^{\mathbf{2}}\right]}\end{array}$ & $\begin{array}{c}\mathbf{V}_{\text {oc }} \\
{[\mathbf{m V}]}\end{array}$ & $\begin{array}{c}\mathbf{F F} \\
{[-]}\end{array}$ & $\begin{array}{c}\text { Efficiency } \\
{[\mathbf{\%}]}\end{array}$ \\
\hline 239 & 41.2 & 656 & 0.771 & 20.9 \\
\hline
\end{tabular}

In addition, we discuss the work in progress: the optimization of the Mercury interconnection design, allowing for industrial module manufacturing with small cell-to-module losses, and our roadmap to develop industrially compatible IBC cells with over $23 \%$ efficiency.

\section{PN-JunCtion ReCOMBINATION}

The surface passivation performance of the FFE, rear emitter and BSF areas can be extracted individually from Sinton quasi-steady state photoconductance (QSSPC) lifetime measurements on test structures with large-area (noninterdigitated) diffusions on each side. The characteristic recombination parameter $J_{0}$ can be determined from the slope of the curve as proposed by Kane and Swanson [5].

\section{A. $J_{0}$ Measurements Of IBC Structured Surface}


The $J_{0}$ determination of surfaces that are typical for an IBC cell, which incorporates emitter and BSF diffusion in an interdigitated pattern, is much more challenging than for a uniformly diffused surface. Both QSSPC or transient photoconductance measurements on these IBC structures are subject to large artefacts that prohibit accurate evaluation of the lifetime and implied $V_{o c}$.

The photoconductance method relies on comparing the conductivity of the sample in dark and under illumination. In dark conditions, the conductivity of the emitter finger of the IBC structure is not measured by the induction coil of the setup, since the pn-junction between emitter and base and also between emitter and BSF fingers are not conductive, as sketched in Fig. 2 left. Thus charges cannot move in and out of this diffused region and its conductivity is neglected.
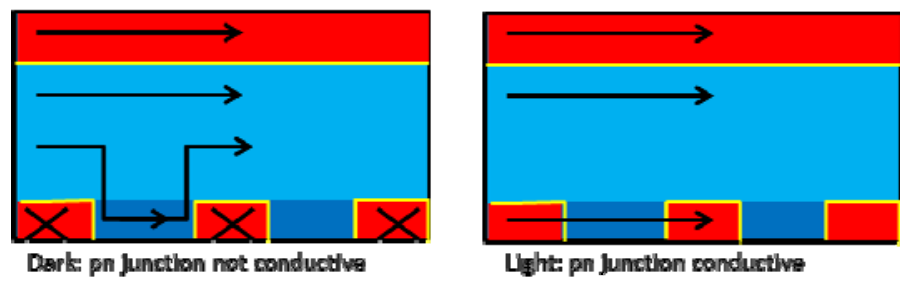

Fig. 2. Schematic overview of conduction paths in dark and light conditions in a cross-section of a Mercury IBC cell. Red and blue correspond to the different polarities of the regions.

However, under illumination the pn-junction starts to conduct as a function of light intensity (injection level) in the sample and the charges can move freely in and out of the diffused emitter (see Fig. 2 right). This increased conductivity is measured by the induction coil. Since the photoconductance method attributes an increase in conductivity to increased carrier concentration in the base of the sample, the enhanced conductivity due to the conducting pn-junctions causes the carrier density to be overestimated.

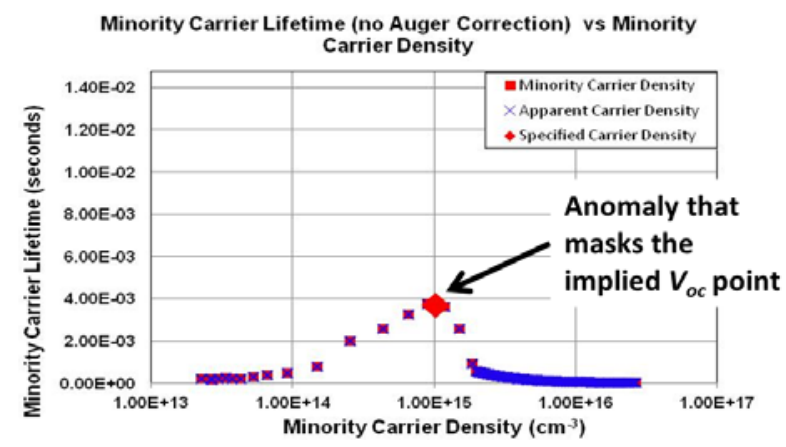

Fig. 3. QSSPC lifetime curve of an IBC cell structure, showing the lifetime anomaly, leading to overestimated implied $V_{o c}$ value.
This results in a sudden increase in apparent lifetime at light intensities that cause the junction to "switch on", leading to an overestimation of the implied $V_{o c}$. This effect on the lifetime curve of an IBC structured sample is shown in Fig. 3, and was reported earlier as well [6].

Despite this effect, the surface $J_{0}$ can still be determined for IBC structures, as it is evaluated at very high injection levels $\left(\Delta n>>10^{15} \mathrm{~cm}^{-3}\right)$ where the lifetime curve is not disturbed by the mentioned artefacts. The linear fit used for this method has a higher off-set but the slope is not affected and can therefore be used to characterize the surface recombination performance of the interdigitated structure.

The recombination parameter $J_{0}$ as determined by the Kane and Swanson method is related to the ideal recombination, i.e. recombination characterized by an ideality factor $\mathrm{n}=1$. This $J_{0}$ can be identified with the $J_{01}$ parameter in a 2-diode equation for the cell. Shockley-Read-Hall recombination occurring in the bulk or in a pn-junction has an ideality factor $\mathrm{n}=2$ and is represented in the diode equation by the $J_{02}$ parameter. In photoconductance measurements of samples with homogeneous diffusions both bulk and pn-junction recombination will be reflected in the effective bulk lifetime.

As explained above, the $J_{02}$ parameter cannot be quantified separately from the effective bulk lifetime value, which is inappropriate when measurements are carried out on IBC patterned samples. The IBC cell architecture that we work with entails a gapless junction between BSF and emitter which could exhibit $J_{02}$ type recombination. As the total pnjunction length that meanders at the rear side of an IBC cell is relatively large, the recombination contribution at the gapless emitter-BSF junction can have considerable impact. In this paper, we present an alternative test method that enables us to quantify the recombination activity at the pn-junctions and confirms that the $J_{0}$ determination from the slope of the QSSPC lifetime curve is insensitive to this type of recombination, as expected.

\section{B. Experimental Set-up}

Test structures were designed with interdigitated emitter and BSF areas on the rear side, and an FFE on the front side. The BSF and emitter fractions were kept constant, while the number of transitions, i.e. pn-junctions, on a test structure was varied between 5 and 20 junctions per centimeter.

Samples with two different rear passivation schemes were prepared, together with samples without any rear passivation. The latter were used as an "extreme" case to illustrate the pnjunction recombination measurement possibilities and limitations, as presented in the next section. The passivation of the FFE was the same in all cases.

The surface passivation of the test structures without rear passivation was evaluated using the Sinton QSSPC lifetime measurements, which excludes the $J_{02}$ and thus the pnjunction recombination contribution in the analysis. The 
resulting $J_{0}$ value is used to calculate the apparent $V_{o c}$ that would be reached if only the recombination at the highly doped surfaces would occur, by using the formula

$$
V_{O C}=\frac{k T}{q} \ln \left(\frac{J_{s c}}{J_{01}}+1\right)
$$

Subsequently, the $V_{o c}$ of the test structure was measured using the Sinton Suns $\mathrm{V}_{o c}$ setup. The $V_{o c}$ measured in this case is affected by all sources of recombination.

From the Suns $\mathrm{V}_{\mathrm{oc}}$ measurement, the $V_{o c}$ and ps- $F F$ are extracted, and both $J_{01}$ and $J_{02}$ are fitted. Subsequently, the dependence of these parameters on the junction density can be evaluated.

\section{Showcase Of Pn-Junction Recombination}

Figure 4 shows the $V_{o c}$ measured in the SunsVoc measurement (blue) and the implied $V_{o c}$ as calculated according to equation (1) from the surface $J_{0}$ values that were determined using the Sinton lifetime tester (red). These values are obtained from a test structure without passivation on the rear, and plotted as a function of the density of pn-junctions on the rear. Note that in both measurements the samples have no metallization. The contacting in the $\operatorname{Suns}_{\text {oc }}$ instrument was done by using metal pins directly in contact with the doped Si surfaces.

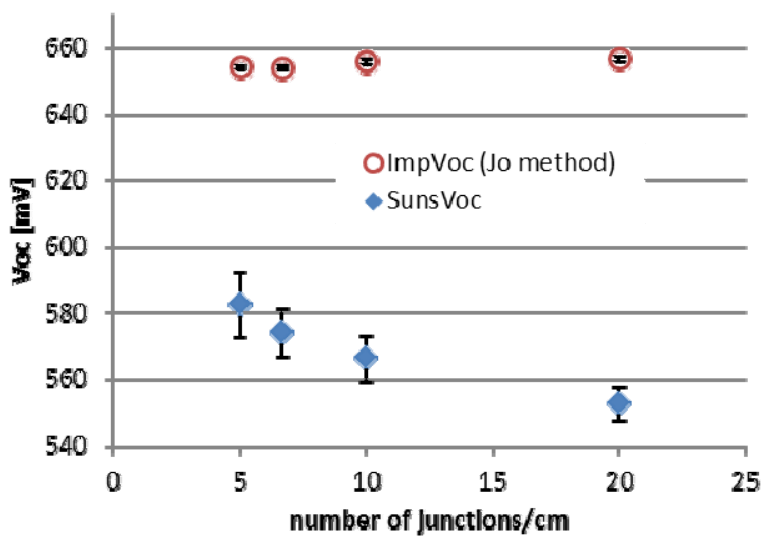

Fig. 4. Implied $V_{o c}$, (derived from the surface $J_{0}$ determination in a Sinton lifetime tester, using equation 1) (red) and $V_{o c}$ measured in a Sinton Suns $\mathrm{V}_{\text {oc }}$ setup (blue), as a function of pn-junction density, for an IBC test structure with no rear passivation.

As can be seen, the $\mathrm{Suns}_{\text {oc }}$ measurement shows a dramatic $V_{o c}$ drop as the junction density increases. The $V_{o c}$ decreases from $\sim 580 \mathrm{mV}$ to $\sim 550 \mathrm{mV}$ as the number of junctions per $\mathrm{cm}$ is increased from 5 to 20 . The implied $V_{o c}$ extracted from the $J_{0}$ determination in the photoconductance measurement instead shows an approximately constant value around $\sim 655$ $\mathrm{mV}$, which is low because of the non-passivated rear side. From these data it is clear that the implied $V_{o c}$ that is calculated from the Sinton lifetime tester data does not take into account the effects of junction recombination, as stated earlier.

\section{Passivated Pn-Junction}

For the experimental investigation of a passivated pnjunction, the doped areas at the rear side were passivated using two passivation layers with different fixed charge density and polarity: NAOS-SiN${ }_{x}$, which is in this paper referred to as $\mathrm{SiN}_{\mathrm{x}}$ passivation (positively fixed charge), and NAOS- $\mathrm{Al}_{2} \mathrm{O}_{3}-\mathrm{SiN}_{\mathrm{x}}$, which is referred to as $\mathrm{Al}_{2} \mathrm{O}_{3}$ passivation (higher negatively fixed charge). NAOS refers to ECN's patented wet-chemical oxidation procedure [7]. On the test structures, metal screen printed fire-through contacts were applied to ensure good contacting during the $V_{o c}$ measurement.

The Suns $V_{\text {oc }}$ data can be used to determine the contribution of pn-junctions to the total recombination losses of the test structure, similar to the method described by Dross et al. [8], and in a way similar to the method described by Fellmeth et al. [9] to determine contact recombination.
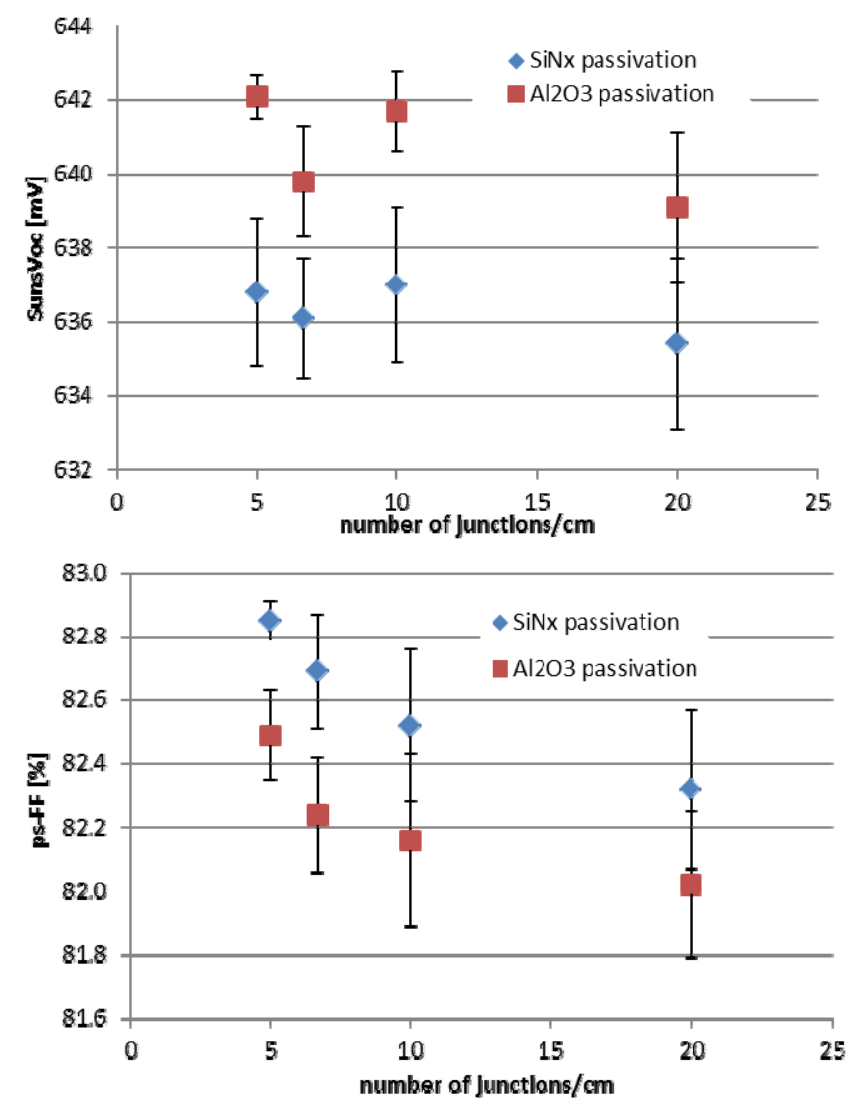

Fig. 5. $\quad V_{o c}$ and pseudo- $F F$ dependence of IBC test structures as a function of pn-junction density. Data are shown for two passivation layers (NAOS-SiN $\mathrm{x}_{\mathrm{x}}$ (blue) and NAOS- $\mathrm{Al}_{2} \mathrm{O}_{3}-\mathrm{SiN}_{\mathrm{x}}($ red)). 
Figure 5 shows a plot of the $V_{o c}$ (top panel) and pseudo- $F F$ (bottom panel), measured by $\operatorname{Suns}_{\mathrm{oc}}$, as a function of the pnjunction density for the samples with the two passivation layers, measured in the center part of the cell. For both passivation cases there is a small and similar decrease of both parameters with increasing number of junctions. Thus no trend difference between the positively and negatively fixed charged layers is observed. The $\mathrm{Al}_{2} \mathrm{O}_{3}$ passivation shows a higher average $V_{o c}$ level due to better passivation of the doped surfaces. However, it also shows a slightly lower pseudo- $F F$ compared to the $\mathrm{SiN}_{\mathrm{x}}$ passivation. The linear dependency of ps- $F F$ with inverse unit cell width, as proposed earlier [8] can be drawn within the error bars.

From the Suns $\mathrm{V}_{\text {oc }}$ measurement, the $J_{02}$ values can be fitted and plotted against the ps- $F F$, as shown in Fig. 6. Note that the fitted $J_{01}$ values were constant for all junction densities (not shown).

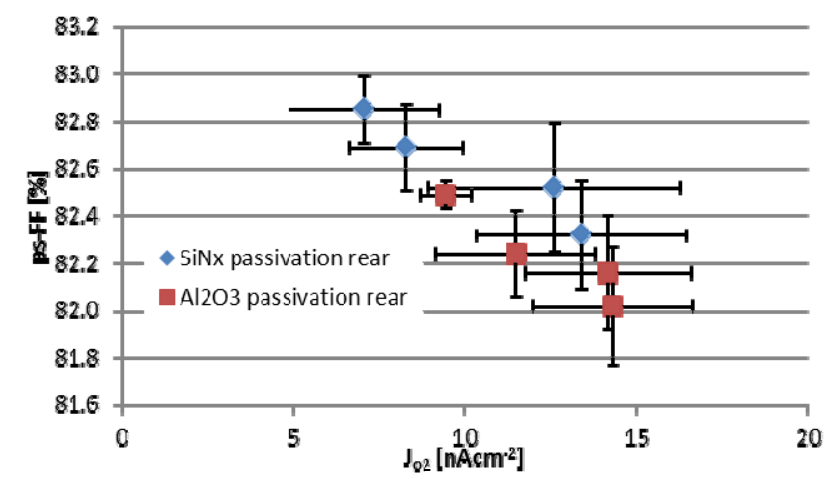

Fig. 6. Pseudo- $F F$ of IBC test structures as a function of fitted $J_{02}$ values in $\mathrm{Suns}_{\mathrm{oc}}$ measurement. Data are shown for two passivation layers (NAOS-SiN ${ }_{x}$ (blue) and NAOS- $\mathrm{Al}_{2} \mathrm{O}_{3}-\mathrm{SiN}_{\mathrm{x}}$ (red).

It is clear from Fig. 6 that the $J_{02}$ and ps- $F F$ data obtained from the test structures with both passivation layers are on the same line. The lower ps- $F F$ (higher $J_{02}$ ) values for $\mathrm{Al}_{2} \mathrm{O}_{3}$ passivation suggests that the $\mathrm{Al}_{2} \mathrm{O}_{3}$ rear-passivated samples suffer from slightly more $J_{02}$ type recombination. As it is not expected that the $\mathrm{Al}_{2} \mathrm{O}_{3}$ passivation layer itself is more defective than the $\mathrm{SiN}_{\mathrm{x}}$ passivation layer (the higher $V_{o c}$ of $\mathrm{Al}_{2} \mathrm{O}_{3}$ passivated samples is indicative for this), there should be another explanation for the lower ps- $F F$. One hypothesis is that the highly negative fixed charges of the $\mathrm{Al}_{2} \mathrm{O}_{3}$ move the actual pn-junction location to a more defective region on the sample, leading to slightly increased $J_{02}$ type recombination.

By taking into account the number of junctions in our current Mercury cell design (approximately 15 junctions $/ \mathrm{cm}$ ), we can estimate from Fig. 5 (using a linear approximation) that the total contribution to $V_{o c}$ and pseudo- $F F$ losses due to recombination at the pn-junction is $0-5 \mathrm{mV}$ and about $0.4 \%$ absolute, respectively. This is a small contribution, especially for these gapless IBC structures. We used a higher metallization fraction in the rear-passivated test structures than currently in the cell, which could slightly underestimate the losses. Although more data are needed to increase the statistical relevance, it is clear that the method presented in this paper can be useful when optimizing the unit cell design and cell passivation.

\section{FUTURE WORK}

\section{A. Cell And Module Design}

ECN has many years of experience in module development for back-contacted solar cells, based on patterned conductive foil. The lay-out of these foil-based modules is very flexible and can be adjusted to any back-contacted cell design. The interconnection design of the Mercury IBC cell as shown here consists of 62 interconnection points to the conducting foil, as shown in Fig. 7. The interconnection points on the cell are contacted to the foil by dots of electrically conductive adhesive, which are printed on the foil and cured during the lamination process of the module.

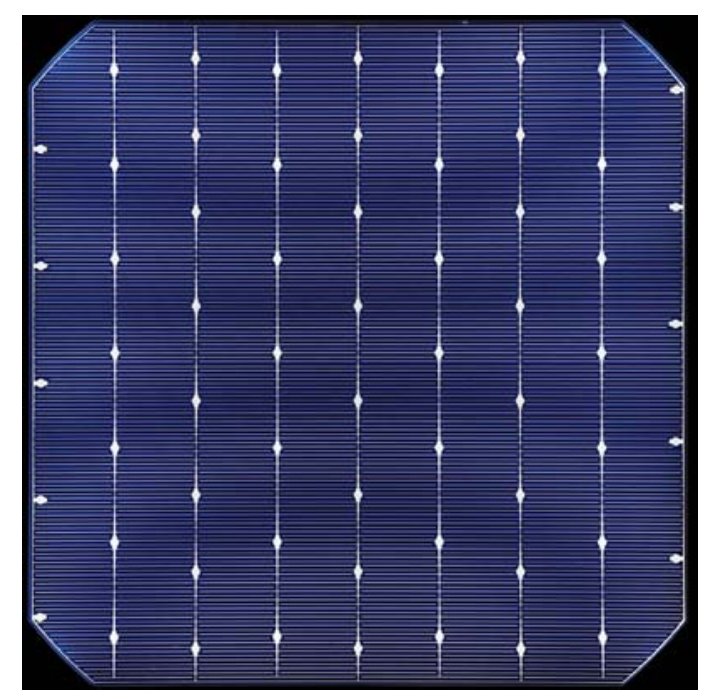

Fig. 7. Interconnection design of the Mercury IBC cell.

Note that the busbars and interconnection points that we use in this design are directly printed and contacted to the cell by fire-through metal paste, just as the fingers. In this way, the relatively wide metal busbar areas are inherently related to wide busbar diffusion areas. Due to the mitigating pumping effect the wider (BSF) diffusion areas will cause only a minor electrical shading loss in the $I_{s c}$ of the cell. However, we do expect some $F F$ losses due to the longer lateral transport path of majority carriers in the bulk at the position of the emitter busbars and interconnection points, which will cause an increase in series resistance. 


\section{$42^{\text {nd }}$ IEEE Photovoltaic Specialists Conference, June 14-19, 2015, New Orleans, USA}

The non-unit-cell related losses, including the ones mentioned above, can be identified in circuit simulations.

\section{B. Cell Process Optimization}

In addition to design optimization, we foresee that process optimization will lead to further efficiency enhancement. In Table IV the efficiency potential of three scenarios is listed. The scenarios are based on 2-D Quokka [10] simulations on a cross-section of a single unit cell (indicated by the dashed box in Figure 1). The resistance of the metal grid (fingers and busbars) are calculated separately, and included as a lumped series resistance in the 2-D Quokka simulation. In these scenarios, several BSF and FFE diffusions and different metallization techniques are considered. We consider for instance standard fire-through (FT) and more gentle non-firethrough (non-FT) metallization. A FT metallization can etch a significant distance $(\sim 200 \mathrm{~nm})$ into the diffusion during firing, resulting in high recombination, especially on light diffusions.

The difference between physical vapor deposition (PVD) and non-FT metallization is not only in the $J_{0}$ values (see Table II), but also in the shape of the contacts. Both require opening of the dielectric prior to application of the metallization. However, the non-FT metallization is assumed to be screen printed, as (narrow) fingers. The PVD metallization is applied full-area, with suitable isolation openings between contacts of opposite polarity.

We considered two geometries: a geometry with BSF and emitter width of 250 and $800 \mu \mathrm{m}$ respectively (abbreviated as $\mathrm{HE}$ ), and one with both BSF and emitter having a width of $1000 \mu \mathrm{m}$ (abbreviated as EM). The different geometries and scenarios result in different metal coverages, as indicated in Table III.

In general somewhat lower efficiencies are expected for the EM geometry, since it has a wider BSF, and will be more prone to electrical shading. However because of the presence of the FFE and the pumping effect, the electrical shading is mitigated to a large extent.

TABLE II

$\mathrm{J}_{0}$ VALUES APPLIED FOR CONTACTED (C) AND NONCONTACTED (NON-C) DIFFUSIONS

\begin{tabular}{|c|c|c|c|c|}
\hline scenario & \multicolumn{2}{|c|}{ BSF } & \multicolumn{2}{c|}{ emitter } \\
\hline & $\mathrm{J}_{0}$ (non-c ) & $\mathrm{J}_{0}(\mathrm{c})$ & $\mathrm{J}_{0}($ non-c) & $\mathrm{J}_{0}(\mathrm{c})$ \\
\hline & $\mathrm{fA} / \mathrm{cm}^{2}$ & $\mathrm{fA} / \mathrm{cm}^{2}$ & $\mathrm{fA} / \mathrm{cm}^{2}$ & $\mathrm{fA} / \mathrm{cm}^{2}$ \\
\hline A & 230 & 1200 & 57 & 2000 \\
\hline B & 72 & 500 & 57 & 450 \\
\hline C & 72 & 500 & 57 & 450 \\
\hline
\end{tabular}

TABLE III

Metal Contact Fractions Values For DifFERENT SCENARIOS AND GEOMETRIES

\begin{tabular}{|l|c|c|c|c|}
\hline & \multicolumn{2}{|c|}{ BSF contact } & \multicolumn{2}{c|}{ emitter contact } \\
\hline scenario & HE & EM & HE & EM \\
\hline
\end{tabular}

\begin{tabular}{|c|c|c|c|c|} 
& $\%$ & $\%$ & $\%$ & $\%$ \\
\hline $\mathrm{A}$ & 7.6 & 4.0 & 4.0 & 3.3 \\
\hline $\mathrm{B}$ & 7.6 & 4.0 & 4.0 & 3.3 \\
\hline $\mathrm{C}$ & 2.9 & 1.5 & 1.5 & 1.5 \\
\hline
\end{tabular}

For our current cell, which features the BSF and metallization scheme according to scenario A, we achieved the cell efficiency of $20.9 \%$ on a full 6 inch wafer, as presented in Table I. However, the difference between the parameters of this cell and of the simulation of scenario A (as shown in Table $\mathrm{V}$ ) is in the $F F$, which we think can in part be explained by non-unit-cell effects.

For the other two scenarios we employed a lighter BSF, which is more difficult to contact using our standard firethrough metallization, but should be well contactable using non-FT metallization in scenario B and PVD metallization in scenario $\mathrm{C}$. These variations were calculated to estimate the losses related to BSF passivation, contact recombination and contact resistance. For example, the emitter and BSF contact recombination in both scenario $\mathrm{B}$ and $\mathrm{C}$ were assumed to decrease from 2000 to $450 \mathrm{fA} / \mathrm{cm}^{2}$ (emitter) and from 1200 to $500 \mathrm{fA} / \mathrm{cm}^{2}$ (BSF), and will therefore boost the efficiency potential to $22.6 \%$ for scenario B and to $23.1 \%$ for scenario C. The optional use of a lighter FFE in scenario $\mathrm{C}$ is expected to give an additional $0.2 \%$ absolute increase in efficiency to $23.3 \%$ (not shown). The sheet resistance of this lighter FFE was $150 \Omega \mathrm{cm}$ while the associated $J_{o}$ was set to $30 \mathrm{fA} / \mathrm{cm}^{2}$. The complete set of IV parameters that resulted from scenario $\mathrm{A}, \mathrm{B}$ and $\mathrm{C}$ is listed in Table $\mathrm{V}$.

TABLE IV

EFFICIENCY POTENTIAL OF MERCURY IBC CELL IN DIFFERENT SCENARIOS $\left(\mathrm{R}_{\text {BULK }}=5 \Omega \mathrm{CM}\right.$, HE GEOMETRY $)$

\begin{tabular}{|c|c|c|c|}
\hline scenario & BSF diffusion & metal & Efficiency [\%] \\
\hline A & heavy & FT & 21.8 \\
\hline B & light & non-FT & 22.6 \\
\hline C & light & PVD & 23.1 \\
\hline
\end{tabular}

TABLE V

IV PARAMETERS OF MERCURY IBC CELL IN DIFFERENT SCENARIOS $\left(\mathrm{R}_{\text {BULK }}=5 \Omega \mathrm{CM}\right)$

\begin{tabular}{|c|c|c|c|c|c|}
\hline scenario & \multicolumn{4}{|c|}{ HE } & EM \\
\hline & $\mathrm{J}_{\mathrm{sc}}$ & $V_{o c}$ & $\mathrm{FF}$ & $\eta$ & $\eta$ \\
\hline & $\mathrm{mA} / \mathrm{cm}^{2}$ & $\mathrm{mV}$ & $\%$ & $\%$ & $\%$ \\
\hline A & 41.2 & 656 & 80.8 & 21.8 & 21.5 \\
\hline B & 41.3 & 672 & 81.6 & 22.6 & 22.4 \\
\hline $\mathrm{C}$ & 41.4 & 680 & 82.3 & 23.1 & 22.9 \\
\hline
\end{tabular}

\section{CONCLUSION}

We presented a method to determine the recombination losses due to the pn-junctions in Mercury IBC cells, and 


\section{$42^{\text {nd }}$ IEEE Photovoltaic Specialists Conference, June 14-19, 2015, New Orleans, USA}

related these losses to the ps- $F F$ of these cells. We conclude from the evaluation of the measurements that pn-junction recombination can be quantified and is especially problematic when the junction passivation is bad. In a linear approximation of the losses associated with the number of pnjunctions, our current cell design does not seem to suffer from large pn-junction recombination losses, possibly due to the excellent level of surface passivation.

We foresee that 23\% Mercury IBC cells are within reach upon changing the BSF diffusion to a lighter profile, and changing the metallization to less recombination-inducing alternatives.

\section{ACKNOWLEDGEMENT}

The authors gratefully acknowledge Ron Sinton for fruitful discussions, and RVO for funding of this research within the Dutch TKI project IBChampion, and the project partners for their collaboration.

\section{REFERENCES}

[1] I. Cesar, N. Guillevin, A.R. Burgers, A.A. Mewe, M. Koppes, J. Anker, L.J. Geerligs, and A.W. Weeber, "Mercury: a back junction back contact front floating emitter cell with novel design for high efficiency and simplified processing" Energy Procedia, vol 55 2014, p. 633

[2] I. Bennett, presented at MWT workshop, 2010, Amsterdam
[3] P. Spinelli, N. Guillevin, T. Burgers, A. Mewe, A. Vlooswijk, B. Geerligs, and I. Cesar, "Front-floating emitter voltage mapping of IBC Mercury cells", SiliconPV, 2015, Constance, to be published.

[4] I. Cesar, N. Guillevin, A.R. Burgers, A.A. Mewe, E.E. Bende, V. Rosca, B. van Aken, M. Koppes, J. Anker, L.J. Geerligs, and A.W. Weeber, "Mercury: a novel design for a back junction back contact cell with front floating emitter for high efficiency and simplified processing" Proc. EUPVSEC, 2014, Amsterdam

[5] D.E. Kane and R.M. Swanson, "Measurement of the emitter saturation current by a contactless photoconductivity method", Proc. $18^{\text {th }}$ IEEE PVSC, 1985, p. 578-581

[6] M. Juhl, C. Chan, M.D. Abbott, and T. Trupke, "Anomalously high lifetimes measured by quasi-steady-state photoconductance in advanced solar cell structures", Applied Physics Letters 103, 2013, 243902

[7] V.D. Mihailetchi, Y. Komatsu, and L.J. Geerligs, "Nitric acid pretreatment for the passivation of boron emitters for n-type base silicon solar cells", Applied Physics Letters 92, 2008, 063510

[8] F. Dross, S. Baker-Finch, K. Fisher, J. Hummel, Y. Lin, P. Murcia, V. Noorai, O. Park, D. Patel, E. Schmich, E. Van Kerschaver, W. Wittmann, and P. Basore, "Quantification of FF losses in p-type screen-printed IBC cell", presented at SiliconPV, 2014, 's-Hertogenbosch

[9] T. Fellmeth, A. Born, A. Kimmerle, F. Clement, D. Biro, and R. Preu, "Recombination at Metal-Emitter Interfaces of Front Contact Technologies for Highly Efficient Silicon Solar Cells, Energy Procedia vol 8, 2011, p. 115

[10] A. Fell, "A free and fast 3D/2D solar cell simulator featuring conductive boundary and quasi-neutrality approximations," IEEE Transactions on Electron Devices, Vol 60 (2), pp. 733$738,2012$. 